\title{
Using Telehealth technology to deliver pulmonary rehabilitation to patients with chronic obstructive pulmonary disease
}

\author{
Michael K Stickland $\mathrm{PhD}^{1,2}$, Tina Jourdain $\mathrm{RRT}^{2}$, Eric $\mathrm{YL}$ Wong $\mathrm{MD}^{1,2}$, Wendy M Rodgers $\mathrm{PhD}^{3}$, \\ Nicholas G Jendzjowsky $\mathrm{MSc}^{3}$, G Fred MacDonald MD²
}

MK Stickland, T Jourdain, EYL Wong, WM Rodgers, NG Jendzjowsky, GF MacDonald. Using Telehealth technology to deliver pulmonary rehabilitation to patients with chronic obstructive pulmonary disease. Can Respir J 2011;18(4):216-220.

BACKGROUND: Pulmonary rehabilitation (PR) is an effective therapeutic strategy to improve health outcomes in patients with chronic obstructive pulmonary disease (COPD); however, there is insufficient PR capacity to service all COPD patients, thus necessitating creative solutions to increase the availability of PR.

OBJECTIVE: To examine the efficacy of PR delivered via Telehealth (Telehealth-PR) compared with PR delivered in person through a standard outpatient hospital-based program (Standard-PR).

METHODS: One hundred forty-seven COPD patients participated in an eight-week rural PR program delivered via Telehealth-PR. Data were compared with a parallel group of 262 COPD patients who attended Standard-PR. Education sessions were administered two days per week via Telehealth, and patients exercised at their satellite centre under direct supervision. Standard-PR patients viewed the same education sessions in person and exercised at the main PR site. The primary outcome measure was change in quality of life as evaluated by the St George's Respiratory Questionnaire (SGRQ). A noninferiority analysis was performed using both intention-to-treat and per-protocol approaches.

RESULTS: Both Telehealth-PR and Standard-PR resulted in clinically and statistically significant improvements in SGRQ scores $(4.5 \pm 0.8 \%$ versus $4.1 \pm 0.6 \% ; \mathrm{P}<0.05$ versus baseline for both groups), and the improvement in SGRQ was not different between the two programs. Similarly, exercise capacity, as assessed by $12 \mathrm{~min}$ walk test, improved equally in both Telehealth-PR and Standard-PR programs $(81 \pm 10 \mathrm{~m}$ versus $82 \pm 10 \mathrm{~m}$; $\mathrm{P}<0.05$ versus baseline for both groups).

CONCLUSION: Telehealth-PR was an effective tool for increasing COPD PR services, and demonstrated improvements in quality of life and exercise capacity comparable with Standard-PR.

Key Words: COPD; Exercise; Pulmonary rehabilitation; Telehealth

\author{
L'utilisation de la technologie de la télésanté pour \\ offrir une réadaptation pulmonaire aux patients \\ ayant une maladie pulmonaire obstructive \\ chronique
}

HISTORIQUE : La réadaptation pulmonaire (RP) est une stratégie thérapeutique efficace pour améliorer les issues de santé chez les patients ayant une maladie pulmonaire obstructive chronique (MPOC). Cependant, la capacité de la RP est insuffisante pour servir tous les patients atteints d'une MPOC, ce qui exige des solutions créatives pour accroître la disponibilité de la RP.

OBJECTIF : Examiner l'efficacité de la RP offerte par télésanté (RP-télésanté) par rapport à la RP offerte en personne au moyen d'un programme ambulatoire en milieu hospitalier (RP-standard).

MÉTHODOLOGIE : Cent quarante-sept patients atteints de MPOC ont participé à un programme de RP-télésanté de huit semaines en milieu rural. Les chercheurs ont comparé les données avec un groupe parallèle de 262 patients atteints de MPOC qui participaient à une RP-standard. Les séances étaient données deux fois par semaine par télésanté, et les patients s'exerçaient à leur centre satellite sous une supervision directe. Les patients recevant la RP-standard avaient droit aux mêmes séances, en salle, et s'exerçaient à l'établissement principal de la RP. La mesure d'issue primaire était un changement de la qualité de vie évalué par le questionnaire respiratoire de St George (SGRQ). Les chercheurs ont effectué une analyse de non-infériorité au moyen des démarches d'intention de traiter et de respect du protocole.

RÉSULTATS : Tant la RP-télésanté que la RP-standard ont suscité des améliorations cliniques statistiquement significatives selon les indices de SGRQ $(4,5 \pm 0,8 \%$ par rapport à 4,1 $\pm 0,6 \%$; $\mathrm{P}<0,05$ par rapport aux données de départ dans les deux groupes). Cependant, il n'y avait pas d'amélioration aux indices de SGRQ entre les deux programmes. De même, la capacité à l'exercice, évaluée par un test à l'effort de 12 min, s'est autant améliorée dans le programme de RP-télésanté que dans celui de RP-standard $(81 \pm 10 \mathrm{~m}$ par rapport à $82 \pm 10 \mathrm{~m} ; \mathrm{P}<0,05$ par rapport aux données de départ dans les deux groupes).

CONCLUSION : La RP-télésanté était un outil efficace pour accroître les services de RP en MPOC et a suscité des améliorations de la qualité de vie et une capacité à l'exercice comparables à la RP-standard.

to rural patients. The purpose of the present study was to examine the efficacy of Telehealth-PR compared with PR delivered in person through a standard outpatient hospital-based program (Standard-PR). It was hypothesized that Telehealth-PR was as effective as Standard-PR at improving quality of life and exercise capacity. while reducing acute exacerbations, hospitalizations and health care costs. In fact, PR has been shown to be a more effective therapeutic strategy for improving health outcomes in patients with COPD than traditional pharmacological management (3-5).

As noted recently (6), there is insufficient PR capacity to serve the COPD population. Furthermore, the majority of PR programs are located within large urban centres, thus limiting access for rural patients. Accordingly, an outpatient PR program delivered via Telehealth technology (Telehealth-PR) was developed to deliver PR
Design and patient selection

The present study was a parallel group, noninferiority trial examining the efficacy of Telehealth-PR compared with delivering 'standard' outpatient hospital-based $\mathrm{PR}$ in person within the main rehabilitation clinic (ie, Standard-PR) at the Centre for Lung Health, Edmonton, Alberta. Enrollment in the study was from January 2006 to December

\section{METHODS}

\footnotetext{
${ }^{1}$ Division of Pulmonary Medicine, Department of Medicine, University of Alberta; ${ }^{2}$ Centre for Lung Health (Covenant Health), Edmonton General Continuing Care Centre; ${ }^{3}$ Faculty of Physical Education and Recreation, University of Alberta, Edmonton, Alberta

Correspondence: Dr Michael K Stickland, Division of Pulmonary Medicine, Department of Medicine, 8334B Aberhart Centre, University of Alberta, Edmonton, Alberta T6G 2J3. Telephone 780-407-7845,fax 780-407-6384, e-mail michael.stickland@ualberta.ca
} 
2008. Patients in the Telehealth-PR group were all referred by their physician to attend Telehealth-PR at their local health centre within nine small communities (population 1000 to 50,000) in central and northern Alberta. Patients within the Standard-PR group were similarly referred by their physician to attend PR at the Centre for Lung Health (Covenant Health), located within Metropolitan Edmonton (population approximately one million). University and hospital research ethics board approval was granted for the present study.

\section{Participants}

All enrolled patients had COPD confirmed by lung function testing and patient history as reviewed by a pulmonologist. Patients included in both groups were deemed suitable for enrollment in PR. Exclusion criteria for both groups were unstable cardiovascular disease and dementia. Patients who were dependent on oxygen or those with comorbidities were included. A total of 147 COPD patients were enrolled in the Telehealth-PR program, while a total of 262 COPD patients were enrolled in the Standard-PR program over the same period. Patient characteristics are presented in Table 1.

\section{Standard-PR}

On referral, all patients were seen by a pulmonologist. Patient history was obtained, baseline lung function and chest $\mathrm{x}$-rays were reviewed and a cardiopulmonary exercise stress test (7) was performed.

The PR program was conducted two days per week for eight weeks. The patients participated in group exercise for $2 \mathrm{~h}$, and attended group education for $1 \mathrm{~h}$ per session. The exercise program was supervised by respiratory therapists or other qualified health professionals, and followed PR guidelines for exercise training (3). Exercise programs included aerobic exercise consisting of track or treadmill walking, cycling and arm ergometer training. The exercise intensity was personalized based on patient symptoms and baseline exercise capacity. Patients performed resistance exercise training that included hand weights and/or elastic bands/tubes. In addition, patients performed flexibility and breathing retraining.

Educational modules directed toward patient self-management (3) were offered for $1 \mathrm{~h}$ each day, with topics including basic pathophysiology of lung disease, pulmonary hygiene, exercise training, respiratory medications, inhaler devices and technique, nutrition, relaxation/ stress management, travel/home care and oxygen therapy.

\section{Telehealth-PR}

Patients referred to the Telehealth-PR program travelled to their local hospital/health clinic and were seen by a pulmonologist at the Centre for Lung Health via Telehealth (ie, video conferencing). Baseline lung function (minimum spirometry), chest x-ray and resting 12-lead electrocardiogram were reviewed by the pulmonologist at the main PR site. The pulmonologist subsequently discussed with the patient their history and symptoms via videoconferencing. The patient was assisted by the respiratory therapist/health professional at the local Telehealth-PR site during their initial consultation.

Patients attended the Telehealth-PR program twice a week for eight weeks within their local community. Identical to Standard-PR, patients performed group exercise for $2 \mathrm{~h}$, and attended group education for $1 \mathrm{~h}$ per session. The exercise program was designed similarly to the main program, with the primary difference being that the main program typically involved eight to 12 patients, while each local PR site had two to six patients. Similar to Standard-PR, the exercise program was supervised by a respiratory therapist or other qualified health professional and included personalized aerobic and resistance exercise training, flexibility exercises and breathing retraining. Local Telehealth-PR staff were given training and guidance from the Standard-PR supervising respiratory therapist ( $T$ Jourdain).

Telehealth-PR patients viewed education sessions that were identical to the main program, delivered to the local sites via video conferencing. At the end of each lecture, patients from all sites were offered the opportunity to ask questions to the main site via Telehealth. The local respiratory therapist/health professional was present at each site to help facilitate program delivery and assist with questions.

\section{TABLE 1}

\section{Baseline patient characteristics}

\begin{tabular}{lcc}
\hline & \multicolumn{2}{c}{ Pulmonary rehabilitation program } \\
\cline { 2 - 3 } & $\begin{array}{c}\text { Standard } \\
(\mathbf{n}=\mathbf{2 6 2})\end{array}$ & $\begin{array}{c}\text { Telehealth } \\
(\mathbf{n}=\mathbf{1 4 7})\end{array}$ \\
\hline Age, years & $69.5 \pm 9.7$ & $69.2 \pm 8.6$ \\
Women/men, $\mathrm{n} / \mathrm{n}(\% / \%)$ & $137 / 125(56 / 44)$ & $69 / 78(47 / 53)$ \\
Weight, kg & $77.0 \pm 22.4$ & $82.5 \pm 21.2$ \\
Height, $\mathrm{m}$ & $164.2 \pm 10.8$ & $168.7 \pm 10.5$ \\
Body mass index, kg/m² & $29.5 \pm 7.7$ & $29.0 \pm 7.1$ \\
Smoking history, pack years & $43.1 \pm 20.2$ & $44.2 \pm 23.3$ \\
Supplemental oxygen therapy, $\mathrm{n}(\%)$ & $71(29)$ & $47(32)$ \\
Comorbid illness, $\mathrm{n}(\%)$ & & \\
Hypertension & $104(42)$ & $56(38)$ \\
Diabetes & $31(13)$ & $14(10)$ \\
Coronary artery disease & $34(14)$ & $25(17)$ \\
Dyslipidemia & $77(31)$ & $35(24)$ \\
Musculoskeletal & $33(14)$ & $27(18)$ \\
\hline
\end{tabular}

Data presented as mean $\pm S D$ unless otherwise indicated. Note: No significant differences were found between the Standard and Telehealth rehabilitation programs

\section{Patient outcomes}

Patients were assessed before and after PR, and all patients were invited to six-month follow-up testing. A priori, the primary outcome measure was set as the change in self-administered St George's Respiratory Questionnaire (SGRQ) total score (8) immediately following PR. A secondary outcome measure was 12 min walk distance. A minimum of two self-paced 12 min walk tests were performed both before and after PR, with mean distance calculated and recorded. A previous study (9) has shown that 12 min walk distance correlates highly $(\mathrm{r}=0.955)$ with 6 min walk distance in patients.

\section{Lung function}

Baseline standardized spirometry was performed on all patients within their community, with patients subsequently stratified according to Global initiative for chronic Obstructive Lung Disease (GOLD) forced expiratory volume in $1 \mathrm{~s}\left(\mathrm{FEV}_{1}\right)$ criteria $(10)$. Where available, lung volume and diffusion capacity were also reported.

\section{Data management and data analysis}

Data obtained for both Standard-PR and Telehealth-PR were all managed centrally in a common database (Windows Access [Microsoft Corporation, USA]). Data were subsequently extracted electronically into a common spreadsheet and verified. It was observed that $13.9 \%$ of scores were missing following PR (T2) compared with baseline (T1), and that $68.4 \%$ of scores were missing at six-month follow-up (T3) compared with baseline. It was, therefore, decided that data imputation to replace missing values at T2 was reasonable, but not for T3. A conservative imputation procedure (last observation carried forward) was used.

A series of $\chi^{2}$ analyses were performed to determine whether there were significant differences between Standard-PR and Telehealth-PR in the incidences of comorbidity, GOLD category, supplemental oxygen and program completion. For analysis purposes, patients were defined as having completed the PR program if they attended a minimum of nine of 16 sessions.

To examine change over time with PR, two analytical strategies were used. First, an intention-to-treat analysis was performed using a repeated measures multiple ANOVA (R-MANOVA) for multiple dependent variables, and R-ANOVA for single dependent variables using the data with replacement (T2 only). Second, a per-protocol analysis was conducted using only those individuals who provided complete data at both T1 and T2, and then again at T3. Due to the high level of attrition at T3 (68\%), only a per-protocol analysis was performed between $\mathrm{T} 1$ and $\mathrm{T} 3$. To evaluate noninferiority between 
TABLE 2

Baseline lung function

\begin{tabular}{|c|c|c|}
\hline & \multicolumn{2}{|c|}{ Pulmonary rehabilitation program } \\
\hline & Standard & Telehealth \\
\hline $\mathrm{FEV}_{1}, \mathrm{~L}$ & $1.24 \pm 0.51$ & $1.23 \pm 0.63$ \\
\hline $\mathrm{FEV}_{1}, \%$ predicted & $48.97 \pm 19.27$ & $48.31 \pm 24.94$ \\
\hline FVC, L & $2.40 \pm 0.77$ & $2.47 \pm 0.98$ \\
\hline FVC, \% predicted & $72.89 \pm 18.96$ & $72.82 \pm 22.90$ \\
\hline $\mathrm{FEV}_{1} / \mathrm{FVC}$ ratio & $0.52 \pm 0.15$ & $0.50 \pm 0.15$ \\
\hline Total lung capacity, L & $5.98 \pm 1.53$ & $5.93 \pm 1.34$ \\
\hline Total lung capacity, \% predicted & $118.43 \pm 28.10$ & $110.06 \pm 21.35$ \\
\hline Residual capacity, L & $4.21 \pm 7.43$ & $3.33 \pm 1.18$ \\
\hline Diffusion capacity, L & $13.19 \pm 4.89$ & $12.15 \pm 4.01$ \\
\hline \multicolumn{3}{|l|}{ GOLD stage, n (\%) } \\
\hline I & $19(7)$ & $11(7)$ \\
\hline II & $95(36)$ & $43(29)$ \\
\hline III & $100(38)$ & $50(34)$ \\
\hline IV & $48(18)$ & $43(29)$ \\
\hline
\end{tabular}

Data presented as mean \pm SD unless otherwise indicated. Note: Spirometry was performed in all patients. Full lung function testing was performed in 235 patients in the Standard program, and in 48 Telehealth program patients.

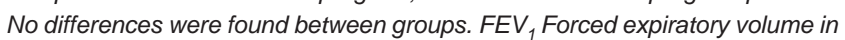
1 s; FVC Forced vital capacity; GOLD Global initiative for chronic Obstructive Lung Disease

programs, a difference of $4 \%$ in SGRQ total score was recognized as the minimum clinically important difference (11) and used to distinguish PR delivered via Telehealth versus Standard-PR. Group data for each variable were expressed as means and SEs. For all inferential analyses, the probability of type I error was set at 0.05 .

\section{RESULTS}

Baseline

No significant difference was observed between Standard-PR and Telehealth-PR in age, weight, body mass index, baseline $\mathrm{FEV}_{1}$, prevalence of comorbidities or use of supplemental oxygen (Tables 1 and 2). The distribution of GOLD stages was also similar in the two programs (Table 2). Despite similarities in $\mathrm{FEV}_{1}$ at baseline, patients in the Telehealth-PR group had higher SGRQ scores in the activities and impacts subscores, and a higher total score (ie, poorer quality of life) compared with Standard-PR. Similarly, distance travelled in the $12 \mathrm{~min}$ walk was less in patients in the Telehealth-PR program at baseline compared with patients undergoing Standard-PR (Table 3).
Program adherence

Patients in the Standard-PR group attended an average of 13.2 PR sessions, while attendance in Telehealth-PR averaged 12.6 PR sessions. No differences were seen in program adherence between the Standard-PR and Telehealth-PR groups (Table 4).

Quality of life (before and after PR)

Using intention-to-treat analysis, an R-MANOVA was conducted using Telehealth-PR versus Standard-PR as the independent variable; the dependent variables were the three SGRQ subscale scores at baseline and program end, with repeated measures for time to control for the baseline scores in the consideration of change. An R-ANOVA was performed separately for SGRQ total score. Both programs demonstrated a clinically and statistically significantly improvement in SGRQ subscale and total scores with PR (Table 4). Despite an improvement in SGRQ score, patients in the Telehealth-PR group still had lower quality of life than Standard-PR after PR. Figure 1 shows that Telehealth-PR was not inferior to Standard-PR because the $95 \%$ CI for the change in SGRQ in both groups was similar and not greater than the prespecified range that defined noninferiority. Similar improvements were observed in symptoms, impact and activity subscales of the SGRQ. Consistent with the intention-to-treat analysis, the per-protocol analysis found that both programs demonstrated a clinically and statistically significant improvement in SGRQ total score with PR, and that the improvement with Telehealth-PR was not inferior to the main program.

12 min walk distance (before and after PR)

An R-ANOVA was conducted using Telehealth-PR versus Standard-PR programs as the between-subjects variable, and 12 min walk distance at each of the three time points as the dependent variable with repeated measures. Both groups increased their walk distances significantly with PR; however, Standard-PR maintained an advantage over Telehealth-PR both before and after PR (Table 3). As shown in Figure 2, the $95 \%$ CI for the difference in 12 min walk distance was similar between groups, indicating that both programs yielded similar improvement in 12 min walk distance. Consistent with the intentionto-treat analysis, the per-protocol analysis showed that individuals in both programs increased their walk distance similarly over time; however, Standard-PR patients walked further than Telehealth-PR patients at both time points.

\section{Six-month follow-up data}

Baseline scores of participants who provided data at six months were compared with those who did not, with no significant differences observed in baseline SGRQ score or walk distance. The improvement in SGRQ total score with PR was maintained at six months, with no

\section{TABLE 3}

St George's Respiratory Questionnaire (SGRQ) and 12 min walk data before, immediately following and six months after pulmonary rehabilitation

\begin{tabular}{|c|c|c|c|c|c|c|c|c|}
\hline & \multicolumn{4}{|c|}{ Standard rehabilitation program } & \multicolumn{4}{|c|}{ Telehealth rehabilitation program } \\
\hline & $\begin{array}{l}\text { Before } \\
(n=262)\end{array}$ & $\begin{array}{c}\text { After } \\
(n=232)\end{array}$ & $\begin{array}{l}\text { After (imputed) } \\
\qquad\left(\mathrm{n}=262^{*}\right)\end{array}$ & $\begin{array}{l}6 \text { months after } \\
(n=88)\end{array}$ & $\begin{array}{l}\text { Before } \\
(n=147)\end{array}$ & $\begin{array}{c}\text { After } \\
(n=121)\end{array}$ & $\begin{array}{l}\text { After (imputed) } \\
\qquad\left(n=147^{*}\right)\end{array}$ & $\begin{array}{c}6 \text { months } \\
\text { after }(n=47)\end{array}$ \\
\hline \multicolumn{9}{|l|}{ SGRQ } \\
\hline Symptoms & $57.9 \pm 20.3$ & $56.3 \pm 19.9^{\dagger}$ & $54.8 \pm 22.0^{\dagger}$ & $46.5 \pm 23.0^{\dagger}$ & $61.4 \pm 18.7$ & $58.8 \pm 18.1$ & $59.6 \pm 17.8^{\dagger}$ & $56.5 \pm 19.1$ \\
\hline Activities & $62.2 \pm 20.2$ & $61.2 \pm 19.6^{\dagger}$ & $58.1 \pm 21.5^{\dagger}$ & $54.9 \pm 23.2$ & $66.9 \pm 19.3^{\ddagger}$ & $61.9 \pm 19.8^{\dagger}$ & $62.7 \pm 20.3^{\dagger \ddagger}$ & $62.7 \pm 22.6$ \\
\hline Impacts & $31.9 \pm 18.5$ & $30.1 \pm 17.8^{\dagger}$ & $27.4 \pm 17.9^{\dagger}$ & $25.8 \pm 17.8^{\dagger}$ & $38.7 \pm 19.4^{\ddagger}$ & $32.1 \pm 17.6^{\dagger \ddagger}$ & $33.6 \pm 18.7^{\dagger \ddagger}$ & $30.0 \pm 14.7^{\dagger}$ \\
\hline \multirow[t]{2}{*}{ Total } & $45.4 \pm 17.1$ & $39.2 \pm 16.4^{\dagger}$ & $41.3 \pm 17.5^{\dagger}$ & $38.1 \pm 18.2^{\dagger}$ & $50.9 \pm 16.2^{\ddagger}$ & $45.5 \pm 16.2^{\dagger \ddagger}$ & $46.8 \pm 16.7^{\dagger \ddagger}$ & $44.6 \pm 15.4^{\dagger}$ \\
\hline & $\begin{array}{l}\text { Before } \\
(n=244)\end{array}$ & $\begin{array}{c}\text { After } \\
(n=237)\end{array}$ & $\begin{array}{l}\text { After (imputed) } \\
\left(n=244^{*}\right)\end{array}$ & $\begin{array}{c}6 \text { months after } \\
(n=128)\end{array}$ & $\begin{array}{l}\text { Before } \\
(n=146)\end{array}$ & $\begin{array}{c}\text { After } \\
(n=121)\end{array}$ & $\begin{array}{l}\text { After (imputed) } \\
\left(n=146^{*}\right)\end{array}$ & $\begin{array}{c}6 \text { months after } \\
(n=35)\end{array}$ \\
\hline 12 min walk & $592.0 \pm 222.7$ & $685.8 \pm 232.1^{\dagger}$ & $689.6 \pm 232.8^{\dagger}$ & $612.7 \pm 214.2$ & $548.6 \pm 242.0^{\ddagger}$ & $665.6 \pm 260.2^{\dagger \ddagger}$ & $630.8 \pm 265.2^{\dagger \ddagger}$ & $621.91 \pm 238.6$ \\
\hline
\end{tabular}

Data presented as mean $\pm S D$. *Missing scores were given the same value as obtained before pulmonary rehabilitation (ie, intention-to-treat analysis). ${ }^{\dagger} P<0.05$ versus before rehabilitation; ${ }^{\ddagger} P<0.05$ versus Standard program 
TABLE 4

Patient dropout during pulmonary rehabilitation in the Standard and Telehealth rehabilitation programs

\begin{tabular}{lcc}
\hline & \multicolumn{2}{c}{ Pulmonary rehabilitation program } \\
\cline { 2 - 3 } & Standard & Telehealth \\
\hline Total & 31 & 20 \\
Respiratory exacerbation & 7 & 6 \\
Hospitalization (other) & 3 & 3 \\
Noncompliant & 4 & 7 \\
Nonrespiratory injury/illness & 6 & 1 \\
Lost contact & 5 & 0 \\
Personal/family issue & 5 & 2 \\
Deceased & 1 & 1
\end{tabular}

Data presented as $n$. Note: No significant differences were found between the Standard and Telehealth rehabilitation programs

between-group difference in SGRQ change (Table 4). At six months, 12 min walk distance was lower compared with immediately following PR. No between-group differences were observed, indicating similar response at six months with Standard-PR and Telehealth-PR.

\section{DISCUSSION}

The purpose of the present study was to examine the efficacy of PR delivered via Telehealth compared with PR delivered in person through a standard outpatient hospital-based program. Telehealth-PR and Standard-PR resulted in similar clinically and statistically significant improvements in quality of life. Similarly, exercise capacity improved equally with Telehealth-PR and Standard-PR. These findings indicate that Telehealth-PR is effective at improving quality of life and exercise capacity in patients with COPD, and provides a viable option to increase capacity and deliver PR services to patients in remote locations who do not have access to PR.

A concern with a noninferiority trial is that the study be sufficiently powered to detect a difference between therapies (12). A difference of $4 \%$ in SGRQ total score is recognized as the minimum clinically important difference for the SGRQ (11), and was used as our evaluation criterion. Previous work from our clinic has shown that the SD of the change in SGRQ with PR is approximately $10 \%$ (13). Using these data, and an alpha level of 0.025 and beta of 0.1 (12), 131 patients in each group would be required to detect a clinically significant difference in SGRQ response between programs. With a sample size of 147 in Telehealth-PR and 262 in Standard-PR, the lack of between-group difference found in our study is unlikely to be explained by insufficient statistical power.

\section{Program components}

The exercise program within both PR programs included aerobic exercise, resistance training, flexibility exercises and breathing retraining. Some of the Telehealth-PR sites had limited equipment, and primarily used walking, as well as simple hand weights and/or elastic bands/tubes for resistance training. Despite the relatively simple approach to exercise, no consistent difference in health outcomes was seen between sites or between the Telehealth-PR and Standard-PR programs. Similarly, recent studies $(14,15)$ have shown that home-based PR is as effective as hospital-based outpatient PR at improving exercise capacity and dyspnea. Home-based and Telehealth-PR approaches can provide effective alternatives to standard outpatient PR. Future studies should examine the efficacy of home-based PR delivered, at least partially, via Telehealth technology as a way of increasing PR access and reducing overall program cost.

\section{Economic considerations}

A recently published economic analysis from our Standard-PR program (16) demonstrated that patients completing PR used less health care resources in the year following PR, resulting in a net reduction of health care costs. Because both Standard-PR and Telehealth-PR demonstrated similar improvements in quality of life and exercise

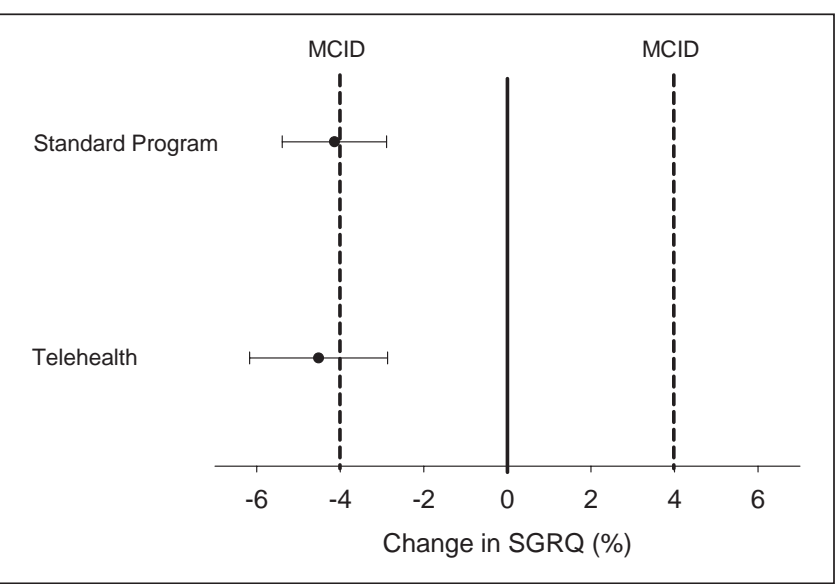

Figure 1) Mean change ( \pm 95\% CI) in St George's Respiratory Questionnaire (SGRQ) with the Standard and Telehealth pulmonary rehabilitation programs. Note: Data presented using intention-to-treat analysis. MCID Minimum clinically important difference

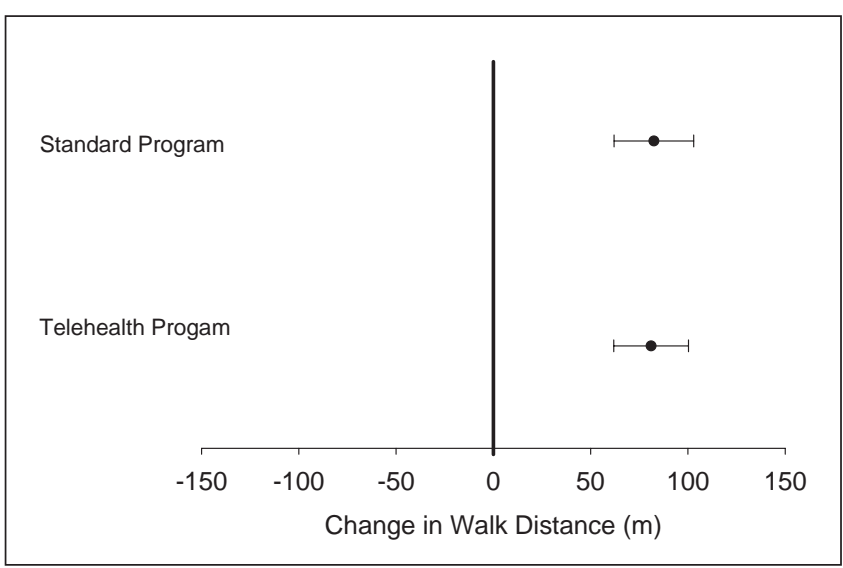

Figure 2) Mean change ( $\pm 95 \mathrm{CI})$ in 12 min walk distance with the Standard and Telehealth pulmonary rehabilitation programs. Data presented using intention-to-treat analysis

capacity, we would expect a similar reduction in health care use in both programs following PR. Telehealth-PR classes were typically conducted with two to six patients, whereas Standard-PR had eight to 12 patients per class; thus, the cost per patient may be higher in Telehealth-PR. However, with central data collection/oversight, as well as one site delivering the lectures, overall costs were likely minimized. Furthermore, we found that Telehealth-PR provided an invaluable support system as remote centres worked to establish PR and COPD management services in their area. Given the positive outcomes of Telehealth-PR, future researchers may wish to specifically examine the economic implications of Telehealth-PR.

\section{Limitations}

Patients in the present study were not randomly assigned to either Standard-PR or Telehealth-PR; thus, it is possible that baseline between-group differences may have biased the results. Some studies have shown that patients with severe COPD improve less with PR compared with patients with less severe disease (17-19). No difference was observed in baseline lung function between our two groups; however, Telehealth-PR demonstrated lower quality of life and exercise capacity at baseline. Based on this, Telehealth-PR may have shown less response to PR compared with Standard-PR; however, we detected no between-group difference in response to PR. These results would indicate that the noninferiority finding was unlikely to be influenced by the lack of patient randomization. 
An additional limitation of the present study was the amount of missing data at six months, which is likely explained by our follow-up procedures. Patients were invited to follow-up either via a telephone call or letter mailed to their home, but were not further encouraged due to funding constraints of the study. Importantly, baseline scores of participants who did provide data at six months were compared with those who did not, and no significant differences were observed in baseline SGRQ scores or walk distance. These findings suggest that patients returning to follow-up at six months in both programs were representative of the original sample. In addition, the primary outcome of the study was the change in SGRQ score immediately after PR, which would not have been affected by missing data at six months. There remains a significant need for stronger evidence regarding the longer-term effects of PR, regardless of mode of delivery.

\section{SUMMARY}

The purpose of the present study was to examine the effectiveness of PR delivered via Telehealth. Telehealth-PR was not inferior to Standard-PR - similar improvements in quality of life and exercise capacity were observed in both programs. Telehealth PR is an effective option to increase PR capacity and deliver PR services to patients in remote locations who do not have access to standard PR.

FUNDING: Funding for this project was obtained from the Alberta Health Services Telehealth Clinical Grant Fund \& Covenant Health Research Foundation.

DISCLOSURE: Dr Stickland is funded by a Canadian Institutes of Health Research New Investigator Award and has received speaking honoraria from GlaxoSmithKline. T Jourdain has no conflicts of interest to disclose. Dr Wong has received speaking honoraria from AstraZeneca, GlaxoSmithKline, Pfizer and Boehringer Ingelheim. Dr Rodgers has no conflicts of interest to disclose. N Jendzjowsky has no conflicts of interest to disclose. Dr MacDonald has no conflicts of interest to disclose.

ACKNOWLEDGEMENTS: The authors thank the participating Telehealth sites and their staff, as well as Drs J Archibald, M Bhutani, A Liu, S Marcushamer, L Melenka, W Ramesh, D Stollery, and the staff at the Centre for Lung Health for their assistance in this project. Funding for this project was obtained from the Alberta Health Services Telehealth Clinical Grant Fund \& Covenant Health Research Foundation. Dr Stickland is funded by a Canadian Institutes of Health Research New Investigator Award.

\section{REFERENCES}

1. Pulmonary rehabilitation: Joint ACCP/AACVPR evidence-based guidelines. ACCP/AACVPR Pulmonary Rehabilitation Guidelines Panel. American College of Chest Physicians. American Association of Cardiovascular and Pulmonary Rehabilitation. Chest 1997;112:1363-96.

2. O'Donnell DE, Aaron S, Bourbeau J, et al. Canadian Thoracic Society recommendations for management of chronic obstructive pulmonary disease - 2007 update. Can Respir J 2007;14(Suppl B):5B-32B.
3. Ries AL, Bauldoff GS, Carlin BW, et al. Pulmonary Rehabilitation: Joint ACCP/AACVPR Evidence-Based Clinical Practice Guidelines. Chest 2007;131:4S-42S.

4. Lacasse Y, Goldstein R, Lasserson TJ, Martin S. Pulmonary rehabilitation for chronic obstructive pulmonary disease. Cochrane Database Syst Rev 2006:CD003793.

5. Lacasse Y, Wong E, Guyatt GH, King D, Cook DJ, Goldstein RS. Meta-analysis of respiratory rehabilitation in chronic obstructive pulmonary disease. Lancet 1996;348:1115-9.

6. Brooks D, Sottana R, Bell B, et al. Characterization of pulmonary rehabilitation programs in Canada in 2005. Can Respir J 2007;14:87-92.

7. ATS/ACCP Statement on cardiopulmonary exercise testing. Am J Respir Crit Care Med 2003;167:211-77.

8. Jones PW, Quirk FH, Baveystock CM, Littlejohns P. A self-complete measure of health status for chronic airflow limitation. The St. George's Respiratory Questionnaire. Am Rev Respir Dis 1992;145:1321-7.

9. Butland RJ, Pang J, Gross ER, Woodcock AA, Geddes DM. Two-, six-, and 12-minute walking tests in respiratory disease. Br Med J (Clin Res Ed) 1982;284:1607-8.

10. Pauwels RA, Buist AS, Calverley PM, Jenkins CR, Hurd SS. Global strategy for the diagnosis, management, and prevention of chronic obstructive pulmonary disease. NHLBI/WHO Global Initiative for Chronic Obstructive Lung Disease (GOLD) Workshop summary. Am J Respir Crit Care Med 2001;163:1256-76.

11. Jones PW. Interpreting thresholds for a clinically significant change in health status in asthma and COPD. Eur Respir J 2002;19:398-404.

12. Jones B, Jarvis P, Lewis JA, Ebbutt AF. Trials to assess equivalence: The importance of rigorous methods. BMJ 1996;313:36-9.

13. Simmonds LG, Rodgers WM, Wong EYL, MacDonald GF, Stickland MK. Patient characteristics that predict completion of pulmonary rehabilitation in COPD. Am J Respir Crit Care Med 2009;179:A5378. (Abst)

14. Guell MR, de Lucas P, Galdiz JB, et al. [Home vs hospital-based pulmonary rehabilitation for patients with chronic obstructive pulmonary disease: A Spanish multicenter trial.] Arch Bronconeumol 2008; 44:512-8.

15. Maltais F, Bourbeau J, Shapiro S, et al. Effects of home-based pulmonary rehabilitation in patients with chronic obstructive pulmonary disease: A randomized trial. Ann Intern Med 2008;149:869-78.

16. Golmohammadi K, Jacobs P, Sin DD. Economic evaluation of a community-based pulmonary rehabilitation program for chronic obstructive pulmonary disease. Lung 2004;182:187-96.

17. Garrod R, Marshall J, Barley E, Jones PW. Predictors of success and failure in pulmonary rehabilitation. Eur Respir J 2006;27:788-94.

18. Wedzicha JA, Bestall JC, Garrod R, Garnham R, Paul EA, Jones PW. Randomized controlled trial of pulmonary rehabilitation in severe chronic obstructive pulmonary disease patients, stratified with the MRC dyspnoea scale. Eur Respir J 1998;12:363-9.

19. Plankeel JF, McMullen B, MacIntyre NR. Exercise outcomes after pulmonary rehabilitation depend on the initial mechanism of exercise limitation among non-oxygen-dependent COPD patients. Chest 2005;127:110-6. 


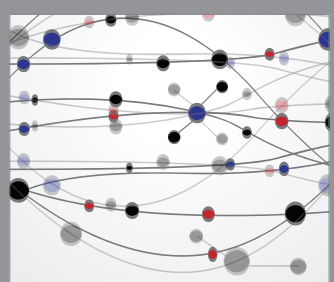

The Scientific World Journal
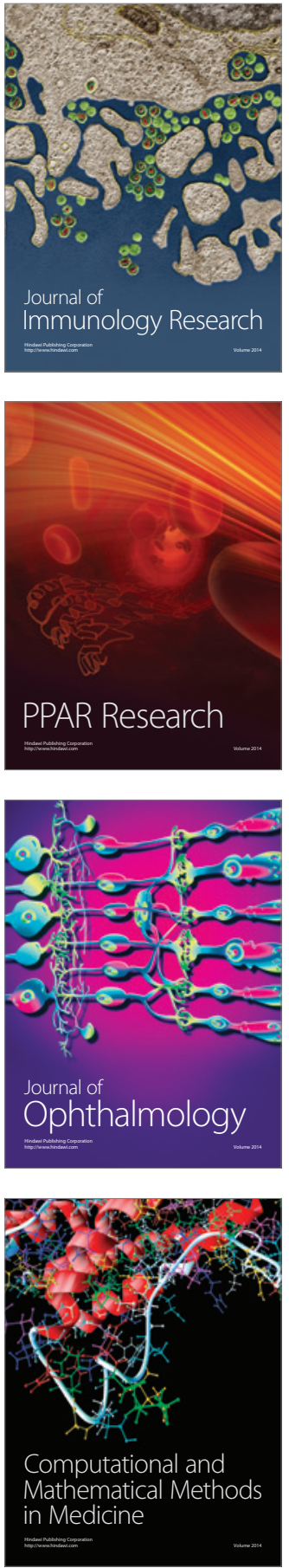

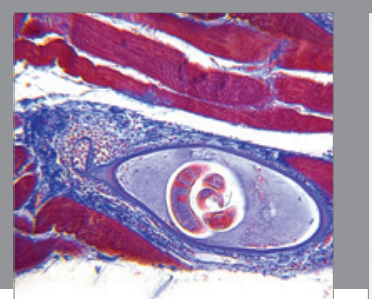

Gastroenterology Research and Practice

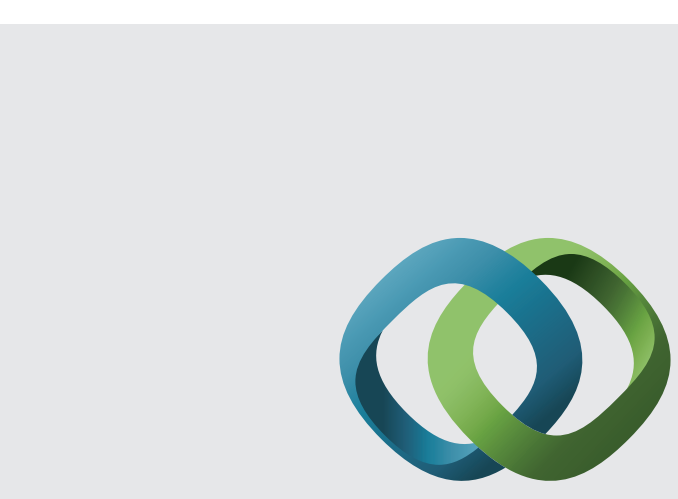

\section{Hindawi}

Submit your manuscripts at

http://www.hindawi.com
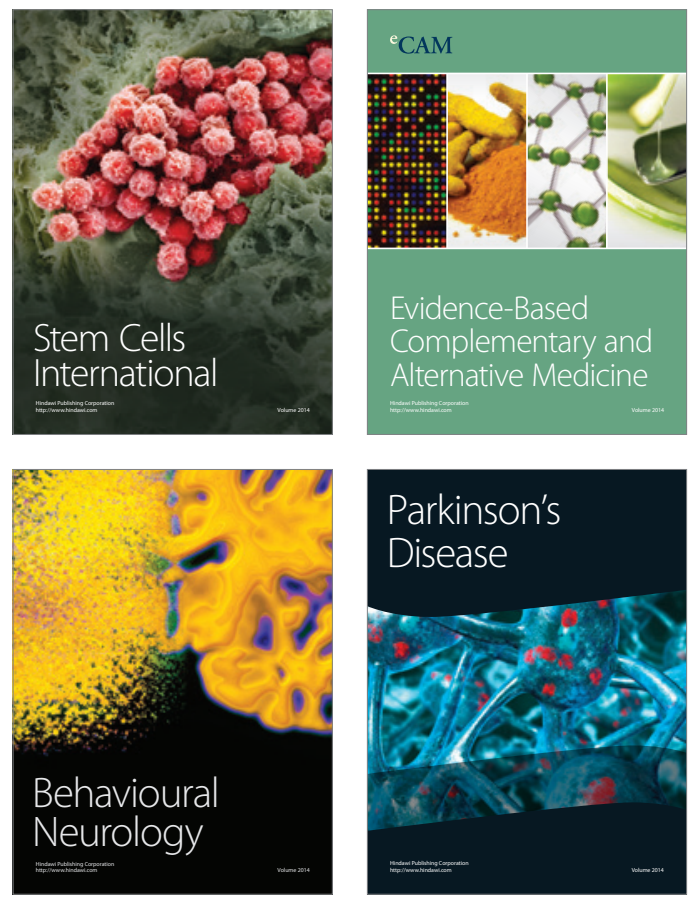
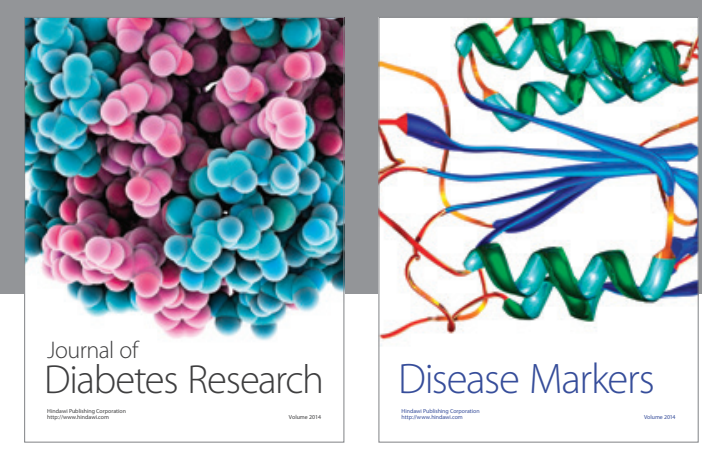

Disease Markers
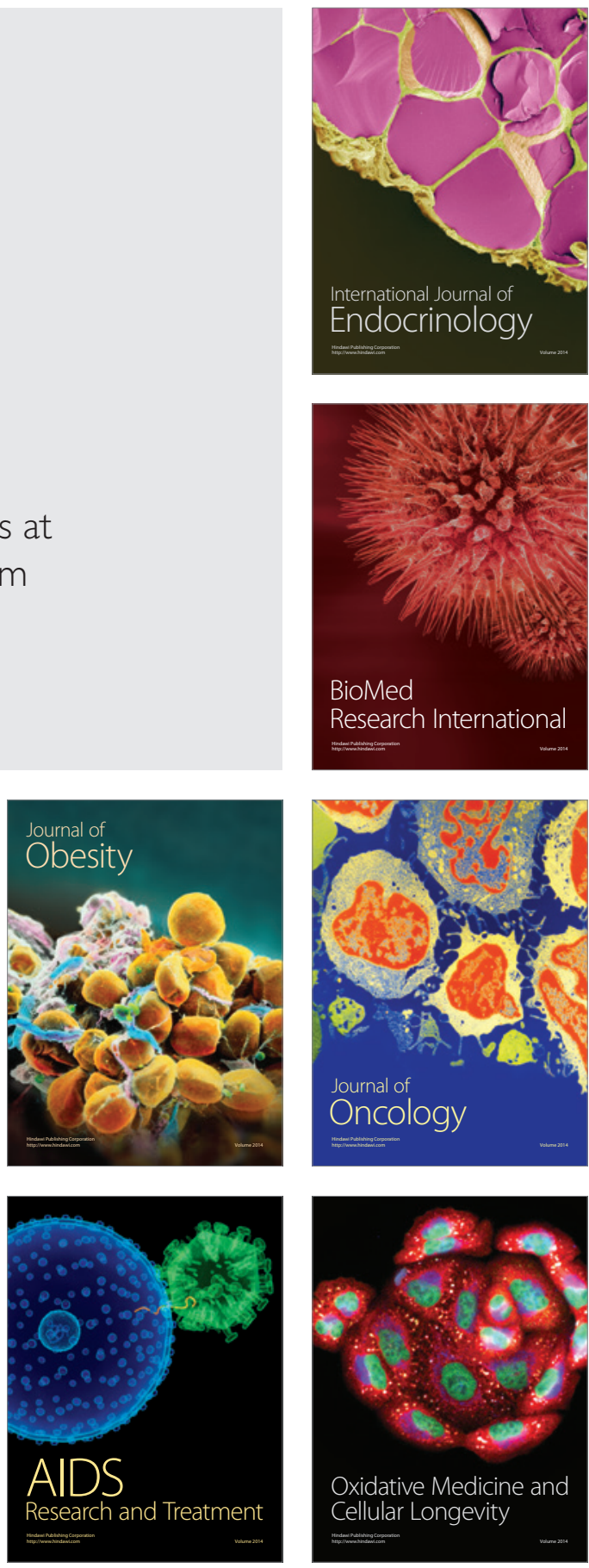